(C) [2009] IEEE. Reprinted, with permission, from [David G. Dorrell, Lucia Frosini, Marcello Bottani, Giacomo Galbiati and Min-Fu-Hsieh, Analysis of Axial Voltages and Inter-bar Currents in Cast Copper Cage Rotors during DC Current Injection as an Aid to Identify Casting Faults, Industrial Electronics, 2009. IECON '09. 35th Annual Conference of IEEE, 3-5 Nov. 2009]. This material is posted here with permission of the IEEE. Such ermission of the IEEE does not in any way imply IEEE endorsement of any of the University of Technology, Sydney's products or services. Internal or personal use of this material is permitted. However, permission to reprint/republish this material for advertising or promotional purposes or for creating new collective works for resale or redistribution must be obtained from the IEEE by writing to pubs-permissions@ieee.org. By choosing to view this document, you agree to all provisions of the copyright laws protecting it 


\title{
Analysis of Axial Voltages and Inter-bar Currents in Cast Copper Cage Rotors during DC Current Injection as an Aid to Identify Casting Faults
}

\author{
David G. Dorrell ${ }^{1}$, Lucia Frosini ${ }^{2}$, Marcello Bottani ${ }^{2}$, Giacomo Galbiati ${ }^{2}$ Min-Fu-Hsieh ${ }^{3}$ \\ ${ }^{1}$ Faculty of Engineering and IT, University of Technology Sydney, Sydney, Australia \\ ${ }^{2}$ Dipartimento di Ingegneria Elettrica, Università di Pavia, Pavia, Italy \\ ${ }^{3}$ Dept. of System and Navel Mechatronic Engineering and Research Center of Ocean Environment and Tech., \\ National Cheng Kung University, Tainan, Taiwan \\ ddorrell@eng.uts.edu.au, lucia@unipv.it,marcello.love@hotmail.it, galbiati.giacomo@gmail.com,mfhsieh@mail.ncku.edu.tw
}

\begin{abstract}
In this paper an investigation is carried out to see if it is possible to assess the condition of a rotor by clamping it in a DCcurrent-injection rig and measuring voltages in bars at regular intervals down the axial length. Experimental measurements are taken on different sections of all the bars for rotors in three different conditions: healthy, with one broken bar at the end, and with all bars broken at each end. A simulation is carried out to compare the results. A complex set of section voltages are found when there are broken bars. There is evidence that this could possibly be a means for testing the condition of rotors during batch manufacturing.
\end{abstract}

\section{INTRODUCTION}

The phenomenon of the inter-bar currents as been discussed for many decades [1], but it is generally disregarded in the classical theory of the induction machines. The usual assumption is that the resistivity of the aluminium (or copper) bars is lower than that of the iron core and the bar-core boundary resistance so that virtually all the current will flow in the cage. The quenching process during manufacture will help increase the bar-core resistance.

As already suggested, the bars of a cage (either fabricated or die-cast) are not insulated from the core, but the bar-tocore boundary represents a high resistance barrier compared to the resistance of the bar. Several experimental studies have proved that current flows between adjacent bars through the core material so that the current distribution along the axial length of the bar is not constant [2]. This is accentuated in skewed cage rotors, since the natural path for the rotor current is down the axial length of the bar, but it is forced along the direction of the skewed bar, as illustrated in Fig. 1.

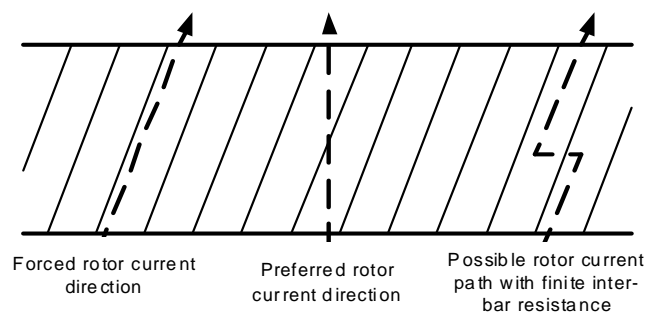

Fig. 1. Preferred and forced current flow in skewed cage rotor.

Inter-bar current become noticeable when there is broken rotor bar [3]-[8]. Several papers have studied how to incorporate the effects of the inter-bar current into induction machine models and simulations in order to correctly predict the behaviour of the motor during its operation with both healthy and faulty rotors. This objective is important for the correct simulation and prediction of the characteristics of the motor for the different applications. This will improve induction machine design procedures (reduction of losses, high starting torque, steady-state performance, etc.) and allow for development of condition monitoring and diagnostics.

In this paper the literature which is related to the effects and analysis of inter-bar current is briefly reviewed. A proposal is then put forward for a method to assess the state of a rotor, particularly small cast rotors, for fractures or casting anomalies. This is supported with experimental results on healthy and faulty rotors in order to analyse the distribution of the current in the bars by measurement of bar voltages down the axial length of the cage when DC current is injected down the length of cage using a non-invasive clamping arrangement. Experimental tests on a rotor with one broken bar provide data about the influence of inter-bar current; the extreme situation of all-broken-bars will highlight that there is still good current paths via the laminations and shaft even with a completely broken set of bars. Some simulation is carried out to assess the voltage behaviour in the assumed rotor circuit.

\section{LITERATURE REVIEW}

According to the classical theory: i) the per-phase models of the induction machine do not take into account the effect of inter-bar current; ii) inter-bar losses are generally included in the stray-load losses; and iii) diagnostics techniques for the detection of broken bars are based on the assumption that a broken bar is insulated from the core and forms an open circuit in the rotor. The existence of inter-bar current has been known for many decades, but it is very complex to model because the inter-bar resistance varies along the axial length and around the periphery of the rotor and its value depends on a number of design, manufacturing and aging factors [9][10].

Nevertheless some recent researchers have proposed models which include the effects of the inter-bar currents and enable torque/speed curve prediction [2][13], steady-state performance [9]-[12] and starting torque [14][15]. Some simulations use equivalent circuits [12]-[14], whereas others employ multi-sliced analytical [2] or multi-sliced 2-D finiteelement solutions [13]-[17]. All the models incorporate different inter-bar circuit configurations using alternative 
radial interconnections. They are implemented using one of the following assumptions [12]:

A) inter-bar current flows only between adjacent bars, straight across the rotor tooth core: the inter-bar resistance is formed by doubling the resistance across the bar-iron (bar-to-core) boundary for a slot side and adding the cross-tooth resistance;

B) inter-bar current flows from one bar to another nonadjacent bar, across a bar-iron contact resistance and through a cross-iron (core) resistance;

C) inter-bar current flow is determined by the bar-iron contact resistance, the core path resistance is negligible.

Each of the above radial circuit configurations leads to an alternative expression for the calculation of the inter-bar resistance parameter from the effective bar-to-bar resistance measured between an adjacent pair of bars. This measurement can be made by means of a method explained in [11] where the end-rings and the last one or two laminations at each end of the stack were removed. A DC current was injected into one bar and excited from an adjacent bar and the bar-to-bar voltage measured. This method assumes that the bar-to-iron contact resistance is uniform along the length of each bar. Its effectiveness has been proved on many cast aluminium cage rotors. Similar methods have been used by others; however this is a destructive test.

For cast or fabricated cage rotors there will be variation due to material properties, manufacturing issues and general aging and wear. For instance, a cast rotor has bar-iron contact down the whole axial length, whereas the fabricated rotor has good contact at the ends of the rotor stacks due to the bar to endring brazing process [15]. Experimental measurements have shown that the inter-bar resistivity is at least a factor of? ten lower in a cast copper rotor compared to a cast aluminium rotor; this has considerable influence on the amplitude of inter-bar currents [18][15].

Broken rotor bars can generate substantial inter-bar current. In larger fabricated rotors, fractures can occur in service due to mechanical and thermal stressing at the ends of the bar. Cast rotors may have air-bubbles or incomplete casting that produces high resistance bar sections. An early study [3] was on large motors with unskewed fabricated copper single-cage machines and subsequently on double-cage motors [4][5]. In these cases the authors have shown theoretically and experimentally that significant inter-bar currents flow when a bar is broken, making invalid the assumption that a broken bar forms an open circuit in the rotor. The currents were measured by means of Rogowski coils placed around the rotor bars between the core and the end-ring. The authors proved that, when the bars were insulated, a relatively low broken bar current flows (about a one third of that expected in a normal bar) and a large increase in the adjacent bar currents occurs to compensate for the missing bar. Moving away from the broken bar, the increased bar current subsides. When the bar is not insulated, the current in the broken bar at the nonfractured end is almost equal to that which flows in a healthy bar; the increase in adjacent bar current is also lower.

Researchers have taken into account this phenomenon in small machines with aluminium [6] or copper (either die-cast or fabricated) [7] cage rotors. The aim was to understand the actual behaviour of an induction motor with a rotor fault, since significant inter-bar currents reduce the magnetic imbalance brought about by a broken bar. This can make the early detection of the rotor fault more difficult, especially when monitoring the sideband currents around the fundamental [8]. In addition, large inter-bar currents will result in large currents flowing in the adjacent bars near the end-ring close to the bar break, causing a chain reaction which could result in the surrounding bar fracturing.

\section{EXPERIMENTAL DC TESTS}

This paper takes a different approach from previous studies. The tests are carried out by injecting a DC current into a complete rotor with the end-rings via clamping plates and measuring the voltage drops in each bar along five axial rotor sections. This is relatively non-invasive. Even in closedslot cast rotors (which are extremely common in small machines) sharp needle probes can be pushed through the bridges over the bars to form good bar contact with little damage since the bridges tend to be less than $1 \mathrm{~mm}$ thick.

The aim of these tests is to analyse the effects of the inter-bar currents on the axial voltages in a cast cage copper rotors in three different conditions: 1) healthy, 2) with one broken bar, 3 ) with all broken bars. These measurements provide an insight into the variation of bar and inter-bar resistance along the axial length of the rotor and into the effects of the broken bars. This allows calculation of a mesh of inter-bar resistances, taking into account that inter-bar current is accentuated in faulty rotors (the mesh is shown in Fig. 2 and described later). In the experimental results below an experimental rig is first investigated using a healthy rotor to assess the reliability of the measurement system. It was then replaced with a similar cast copper cage which had one bar drilled out at one end in order to break it. After this, the broken-bar rotor was further modified by the drilling of every bar at alternate ends. Both the healthy and faulty cage rotors were die-cast with copper (see Table I and Fig. 3).

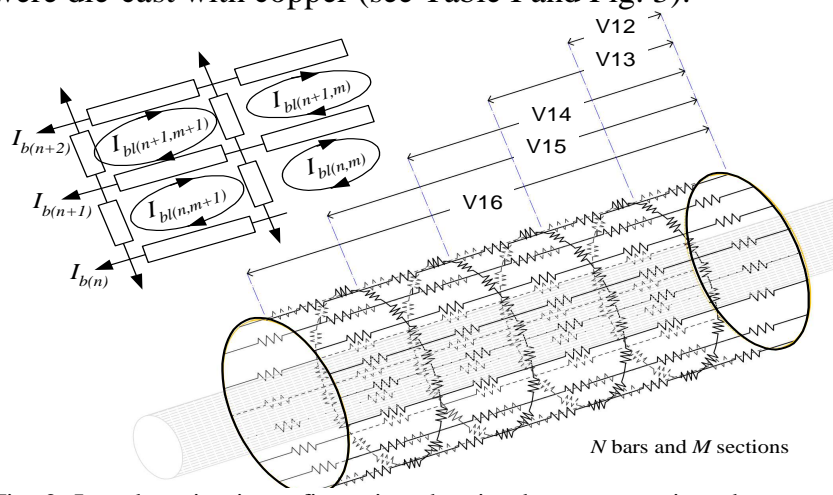

Fig. 2. Inter-bar circuit configuration showing bar currents, inter-bar current loops and resistance mesh.

\section{A. DC tests on the healthy rotor}

To assess the experimental arrangement a healthy rotor was first used. The tests were carried out by injecting DC current in the rotor by means of a test rig. Copper plates were clamped around the end-rings and tightened securely to get good injection connection (Fig. 4). The current flows through these plates into the end-rings and down the rotor bars. It was 
necessary to uncover the bars because the tests require a good contact between copper and the probes. As discussed above, sharp probes could be used as an alternative which can poke through the bar bridge. The magnitudes of the injected current during the tests were $100 \mathrm{~A}, 150 \mathrm{~A}$ and $200 \mathrm{~A}$. The current density in each bar remains quite low (about $0.2 \mathrm{~A} / \mathrm{mm}^{2}$ even for $200 \mathrm{~A}$ ) which is the maximum current value available from the DC supply; hence rotor overheating is not an issue (each test requires about 1 minute).

TABLE I

MAIN CHARACTERISTICS OF THE ROTORS

\begin{tabular}{|l|l|l|l|}
\hline Copper resistivity & $0.0178 \times 10^{-6} \Omega \mathrm{m}$ & Stack length & $180 \mathrm{~mm}$ \\
\hline Rotor diameter & $93.1 \mathrm{~mm}$ & Shaft diameter & $16 \mathrm{~mm}$ \\
\hline Bar number $\left(\mathrm{N}_{\mathrm{b}}\right)$ & 16 & Pole number & 2 \\
\hline Bar depth & $11 \mathrm{~mm}$ & Tooth width & $2.879 \mathrm{~mm}$ \\
\hline Slot area & $52.6928 \mathrm{~mm}^{2}$ & Bar area $(\mathrm{S})$ & $47.15 \mathrm{~mm}^{2}$ \\
\hline Skew & 1.3333 & Lam. thickness & $0.65 \mathrm{~mm}$ \\
\hline
\end{tabular}

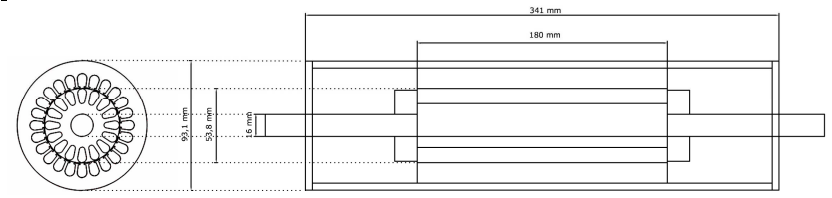

Fig. 3. Motor cross and axial sections.

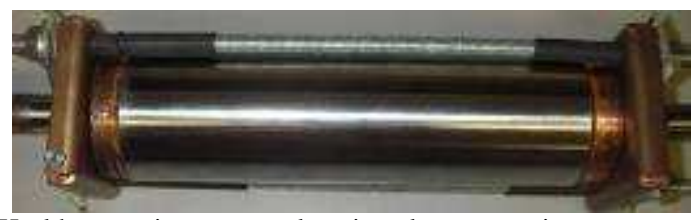

Fig. 4. Healthy rotor in a copper clamping plate connection.

The voltage drops in each section are shown in Table II and 5. The slight variation in section voltages $V_{12}$ and $V_{56}$ are due to the position of the probes at the points 1 and 6 , as shown in Fig. 6; they are located on the end-rings where the copper section is considerably bigger than the bar section and hence the voltage drop is lower due to lower resistance of the rings.

The bar resistance of each section can be calculated using

$$
R=N_{b} V / I
$$

Where the bar number $N_{b}=16$ is the bar number and the bars are nominally identical, so that the current is split equally between the bars. These experimental resistance values are shown in Table III, where it can be noticed that the values obtained in the three tests are similar. The theoretical value of each bar resistance $\left(R_{16}\right)$ is close to the experimental values:

$$
R=\frac{\rho \cdot l}{S}=\frac{0.0178 \cdot 0.180}{52.6928}=60.80 \mu \Omega
$$

VOLTAGE DROP ON A BAR OF THE HEALTHY ROTOR

\begin{tabular}{|c|c|c|c|}
\hline & $100[\mathrm{~A}]$ & $150[\mathrm{~A}]$ & $200[\mathrm{~A}]$ \\
\hline $\mathrm{V}_{12}[\mathrm{mV}]$ & 0.047 & 0.066 & 0.095 \\
\hline $\mathrm{V}_{23}[\mathrm{mV}]$ & 0.078 & 0.118 & 0.158 \\
\hline $\mathrm{V}_{34}[\mathrm{mV}]$ & 0.079 & 0.124 & 0.166 \\
\hline $\mathrm{V}_{45}[\mathrm{mV}]$ & 0.080 & 0.117 & 0.161 \\
\hline $\mathrm{V}_{56}[\mathrm{mV}]$ & 0.063 & 0.095 & 0.124 \\
\hline
\end{tabular}

\section{B. DC tests on the one-broken bar rotor}

The broken-bar rotor has one bar drilled out at one end (left, Fig. 6) and each bar was split into five sections where it was possible to see the bar surface for good connection. The voltage drop at each section for all 16 rotors bars was measured by attaching a fixed probe at point 1 and the second probe at every point up to point 6 (see Fig. 6).

TABLE III

RESISTANCE VALUE IN EACH SECTION OF A BAR OF THE HEALTHY ROTOR CALCULATED FOR EACH VALUE OF INJECTED CURRENT

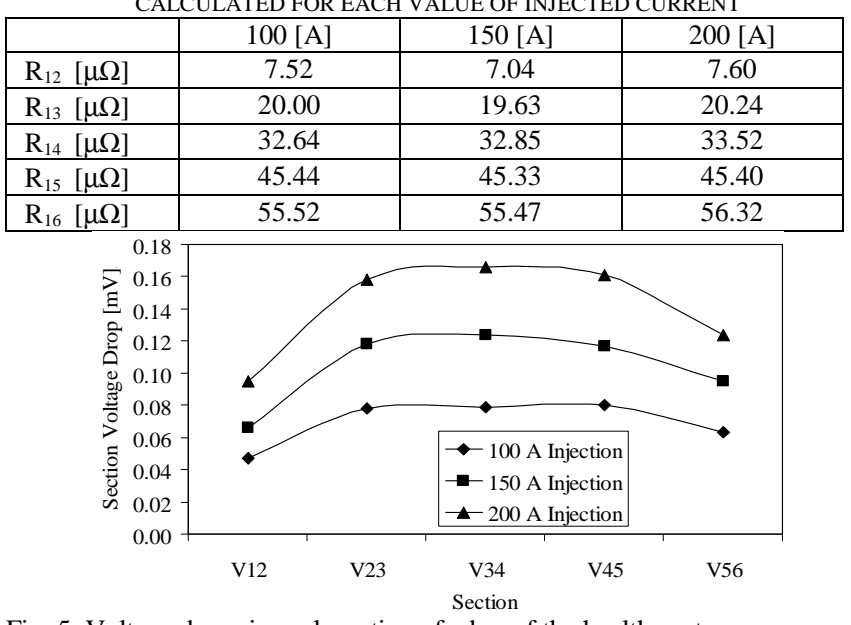

Fig. 5. Voltage drops in each section of a bar of the healthy rotor.

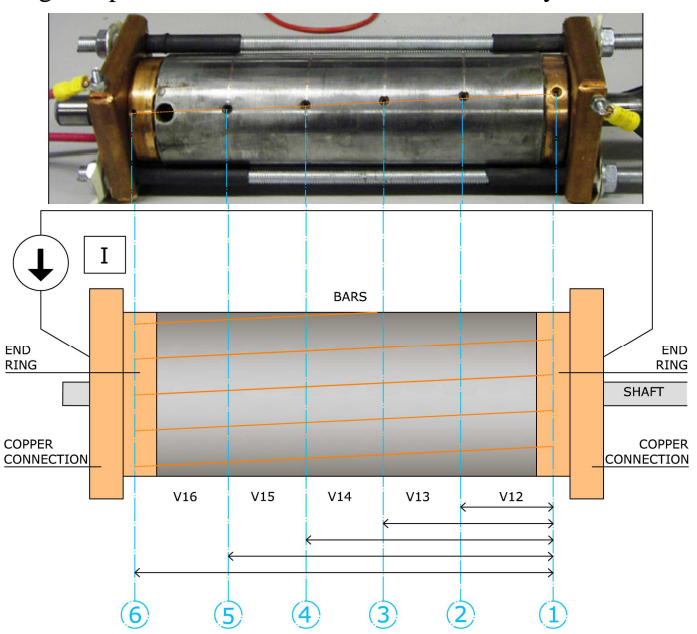

Fig. 6. Rotor with one broken bar split in 5 sections.

The tests were carried out at 100, 150 and $200 \mathrm{~A}$, as shown in Fig. 7. The section voltage drops along the broken bar were not uniform along the bar; the three lines have the same characteristic, illustrating consistency in the tests. It is possible to compare the voltage drops along the broken bar with the voltage drops along other bars, in particular with the adjacent bars and the opposite bar. Figs. 8 and 9 compare the broken bar and the adjacent bar voltages. It can be seen that in the adjacent bars the voltage drops are almost constant in the central sections, with lower voltages in the end sections; however, the overall lines are almost straight.

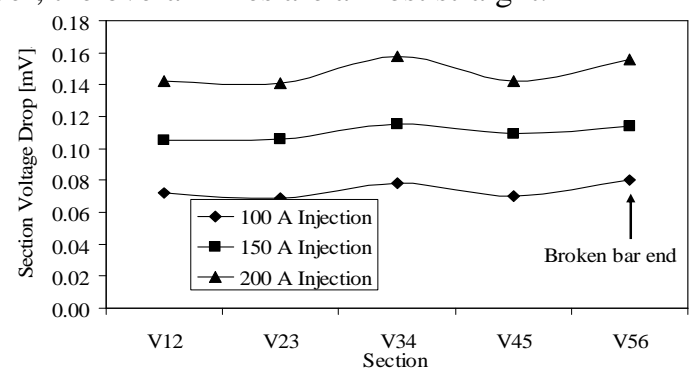

Fig. 7. Voltage drops in each section of the broken bar. 
There is a different behaviour at both ends of the opposite bar (Fig. 10). In all three cases (100, 150 and 200A) there is a small decrease in V12 however there is a more pronounced decrease in the V56, where the bar is broken while there is an increase in V56 for the broken bar. This is due to a redistribution of the currents in the bars caused by the fault.

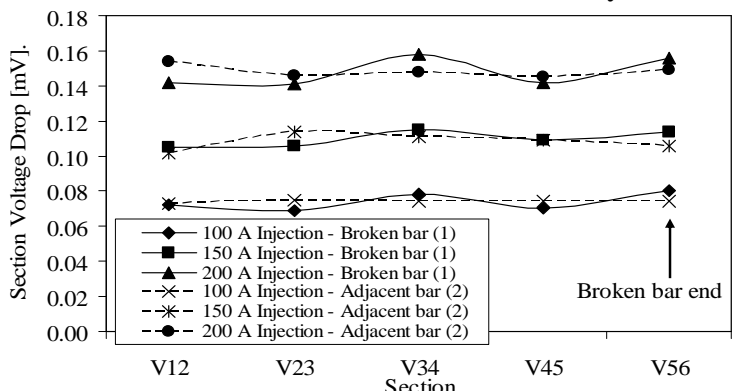

Fig. 8. Comparison between the broken bar and the adjacent bar 2 (up).

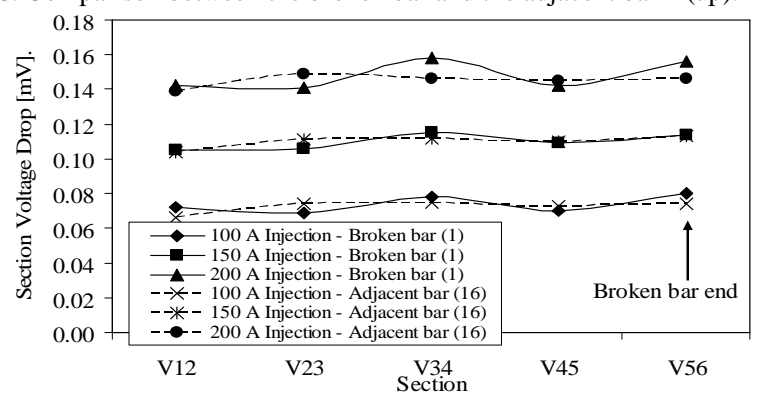

Fig. 9. Comparison between the broken bar and the adjacent bar 16 (down).

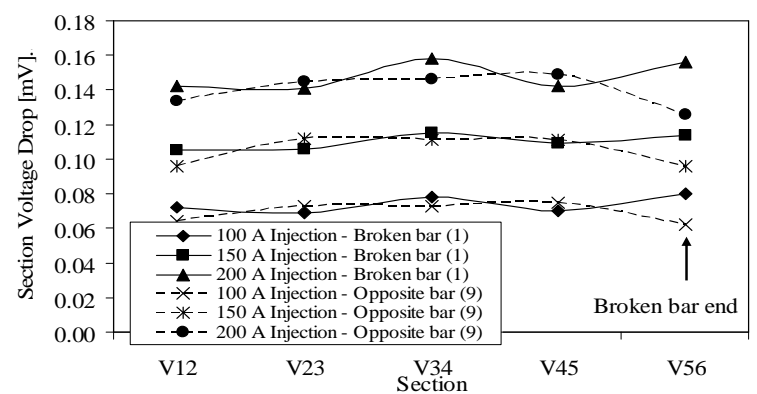

Fig. 10. Comparison between the broken bar and the opposite bar 9.

The section voltage drop trends in the healthy bars of the faulty rotor can compared with the voltage drops in the bars of the healthy rotor; the two rotors are different, but of the same design. In Fig. 11 it can be seen that the voltages of the healthy rotor is very similar to that of the opposite bar of the one-broken-bar rotor. The difference between the two cases is probably due to fabrication differences.

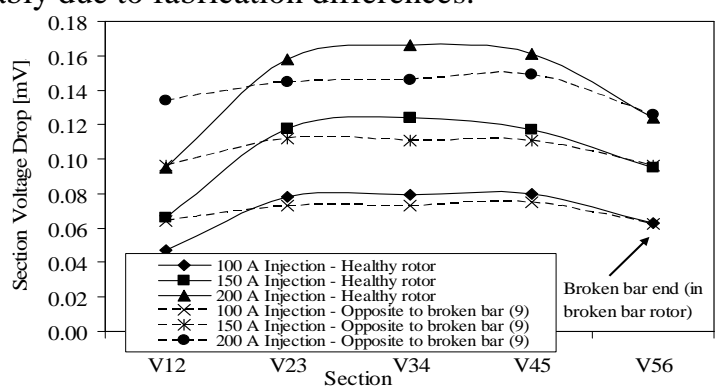

Fig. 11. Comparison between the opposite bar of the one-broken-bar rotor and the bar of the healthy rotor.

The voltage drop trends for all the bars of the faulty rotor can be investigated. Three different kinds of trend can be observed: i) constant (Fig. 12); ii) "concave" (Fig. 13); and iii) "convex" (similar to the healthy rotor - Fig. 14). In these figures the 200 A characteristics only are shown for clarity.

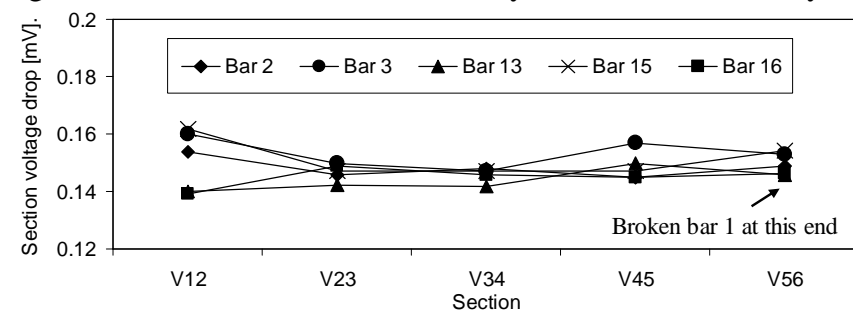

Fig. 12. Bars with constant trend (200 A injected) - one broken bar rotor.

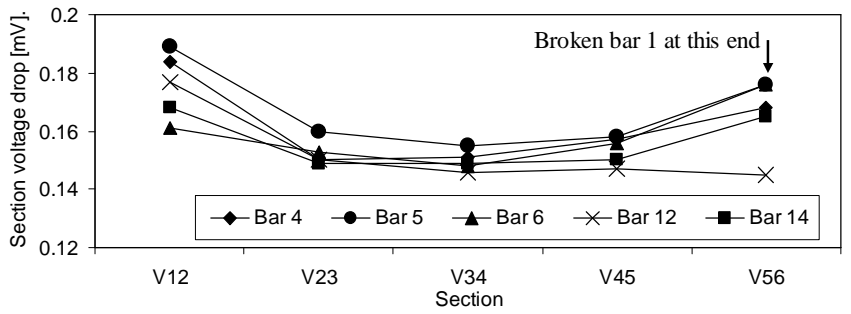

Fig. 13. Bars with concave trend (200 A injected) - one broken bar rotor.

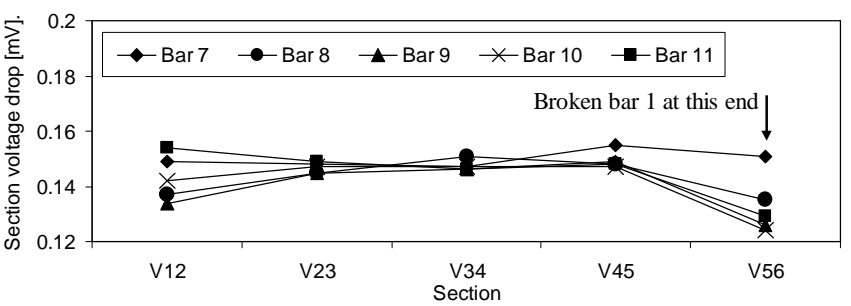

Fig. 14. Bars with convex trend (200 A injected) - one broken bar rotor.

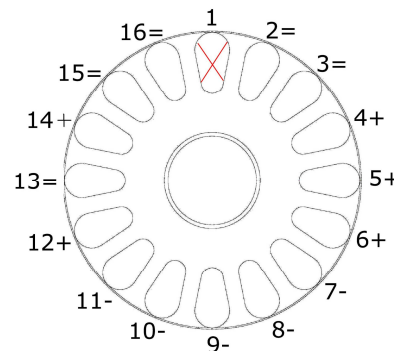

Fig. 15. Bars affected by concave trend (+), convex (-) and constant (=) showing some symmetry between left and right hlaves (except for 13 and 5 which could be do casting variation).

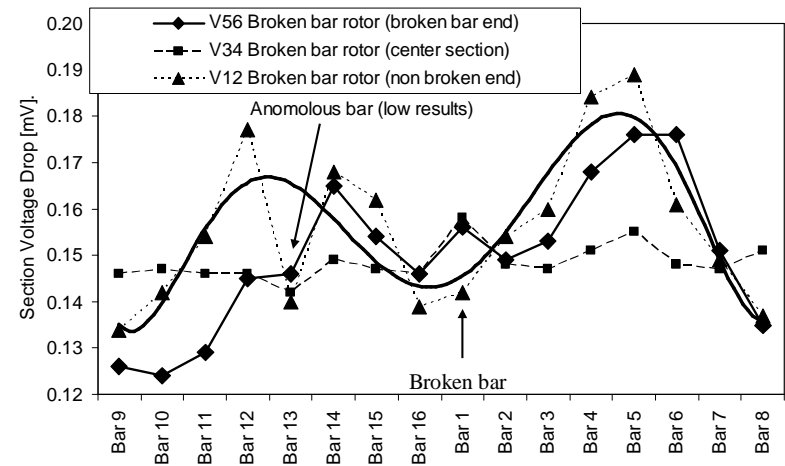

Fig. 16. Voltage drops on all the bars of the one-broken-bar rotor (200 A injected) including $6^{\text {th }}$ order polynomial trend line for V56 (broken bar end).

In summary, in the one-broken-bar-rotor, these results seem to show that the bar section voltages are affected by the failure. By inspection of the bar section voltages over all the 
bars it should be possible to identify broken bars and rotor cage anomalies by characteristic section voltage patterns. The "constant" and "concave" shapes depend on the inter-bar currents which pass through the iron core into the other bars. Fig. 15 shows the relative change in magnitude of $V_{56}$ (increase or decrease) for all the bars. In Fig. 16, $V_{12}, V_{34}$ and $\mathrm{V}_{56}$ are given, it is clear that the broken bar appears to give an oscillatory form to the bar voltages of the end sections, which indicates the nature of the distinctive voltage patterns that characterize a broken bar. Work is needed to validate the patterns over a number of production rotors with faults.

\section{DC tests on the all-broken bars rotor}

The rotor with one broken bar was modified by drilling out each rotor bar at alternate ends, as shown in Fig. 17. The same tests illustrated in the previous section were repeated with the rotor in this condition.

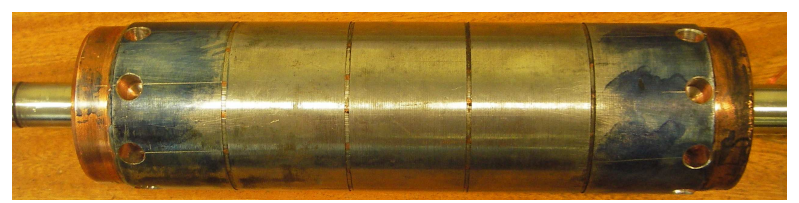

Fig. 17. Rotor with all broken bars split in 5 sections.

The curves of the voltage drops measured with current injection present the same "concave" trend. It follows that all the bars have increased $\mathrm{V}_{12}$ and $\mathrm{V}_{56}$. The similar characteristics are due to the faulty but electrically symmetrical condition of the rotor. Fig. 18 shows the curves for $\mathrm{V}_{12}, \mathrm{~V}_{34}$ and $\mathrm{V}_{56}$ for $200 \mathrm{~A}$ injection across all the bars.

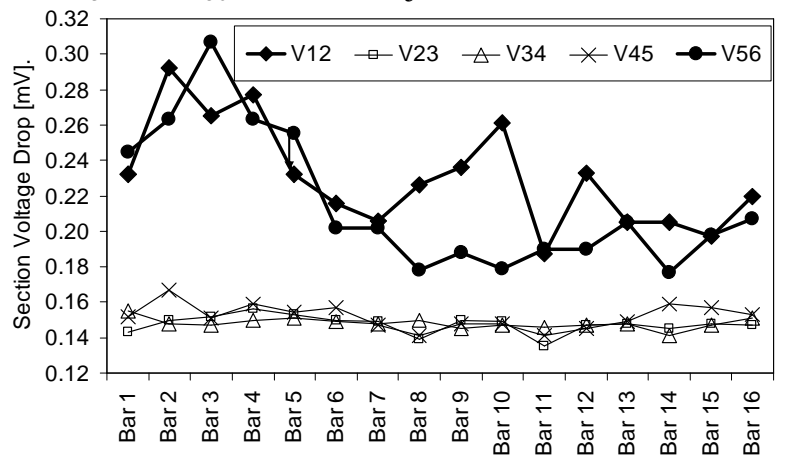

Fig. 18. Voltage drop in each section of the bars of the all-broken-bar rotor (200 A injected).

Another interesting consideration can be drawn by analysing Fig. 19 which shows the voltage drops in the healthy bar in the healthy rotor, broken bar in the one-brokenbar rotor and broken bar in the all-broken-bars rotor. For V12 and V56 there are three trends: i) reduced voltage drop for the healthy rotor; ii) almost constant voltage drop for one broken bar; and iii) increased voltage drop for the rotor with all broken bars. By comparing the behaviour of the all-brokenbars rotor with the healthy one, it can be seen that the endsection voltages increase when all the bars are broken as expected. While only alternate bar ends are broken at each end, this still increases the end-section voltages in the adjacent the unbroken bars since these bar sections will be carrying higher current. In comparison with the one-brokenbar case, where the inter-bars currents affect bars close to the broken bar, and produced a oscillating section voltage characteristic (Fig. 16), the end-section voltage drops are all increased although there is some inconsistency in the magnitudes which may be due to fabrication and modification (bar drilling) variations.

It can be concluded that the effects of inter-bar current are complex. As already stated, predicting the section voltage variation with broken or incomplete bars is not straightforward and should be further investigated in a number of rotors with different faults.

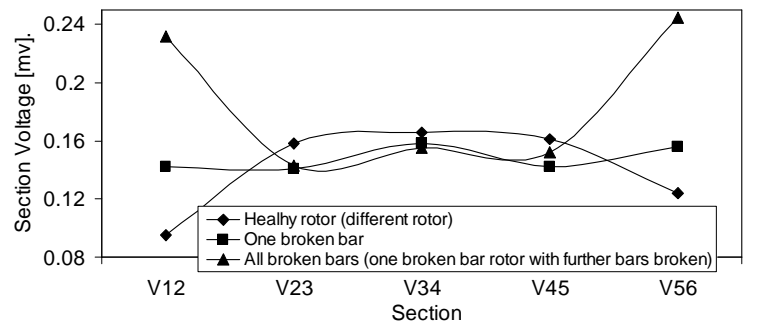

Fig. 19. Comparison among healthy rotor, one-broken-bar rotor and allbroken-bars rotor (200 A injected).

\section{SIMULATION}

The circuits required for obtaining the inter-bar resistance and integrating it into a machine model is complex [2][6][11] [15]. It can be seen from the results that even breaking the bar completely does not prevent current flowing down the bar with circumferential paths available to other bars and also axial paths are available along incomplete lamination insulation (e.g., at edges where there is burr) and the shaft. However a simple DC model of the rotor with one broken bar can be implemented (Fig. 2). For simplicity it is assumed that there is no axial current path through the laminations and shaft. Therefore this model is only appropriate for one (or very few) broken bars. The circuit was simulated in MATLAB. While the voltage is calculated at six axial points (five sections), fifty sections were used in the simulation with the voltage calculated every ten sections. This accounts for the distributed nature of the inter-bar resistance along the length of the bars. It was decided to simulate the circuit using a bar current in each bar and inter-bar currents as shown in Fig. 2. Hence, for a driving end-ring-to-end-ring voltage $V$, the impedance matrix is:

$$
\left[\begin{array}{l}
V \\
\cdots \\
V \\
0 \\
\cdots \\
0
\end{array}\right]=\left[\boldsymbol{R}_{\text {matrix }}\left[\begin{array}{l}
I_{b(1)} \\
\cdots \\
I_{b(N)} \\
I_{b l(1)} \\
\cdots \\
I_{b l(N \times M)}
\end{array}\right]\right.
$$

Each resistance component can be set and a resistance matrix formulated. The bar currents were driven by the clamping voltage (which was adjusted to get an input current of $200 \mathrm{~A}$ ). The bar resistance was calculated from the machine geometry and the resistivity of copper $\left(20^{\circ} \mathrm{C}\right)$. The inter-bar resistance could be varied and a broken bar inserted by inclusion of a high resistance element in one end section. The results are shown in Fig. 20 for the cases of complete rotor (Case 1) and 
one broken bar (with high inter-bar resistance and broken bar - Case 2, and with low inter-bar resistance and high resistance (partially broken) bar end section - Case 3). It can be seen that Cases 1 and 2 show expected results with identical voltage in the sections apart from the bar which is broken where there is a much high voltage drop across V56. However when there is a high resistance break rather than complete break, and the inter-bar resistance is much lower (as appears to be the case here in the experiments - Fig. 16) then there is a complex set of section voltages which show oscillation. While it does not exactly match Fig. 16, it should be borne in mind that the axial resistance paths through the laminations and shaft are not included in the circuit and the simulation is meant to be more indicative rather than precise. It is therefore suggested that there is evidence that this sort of DC current injection could be used to identify casting issues in cage rotors.

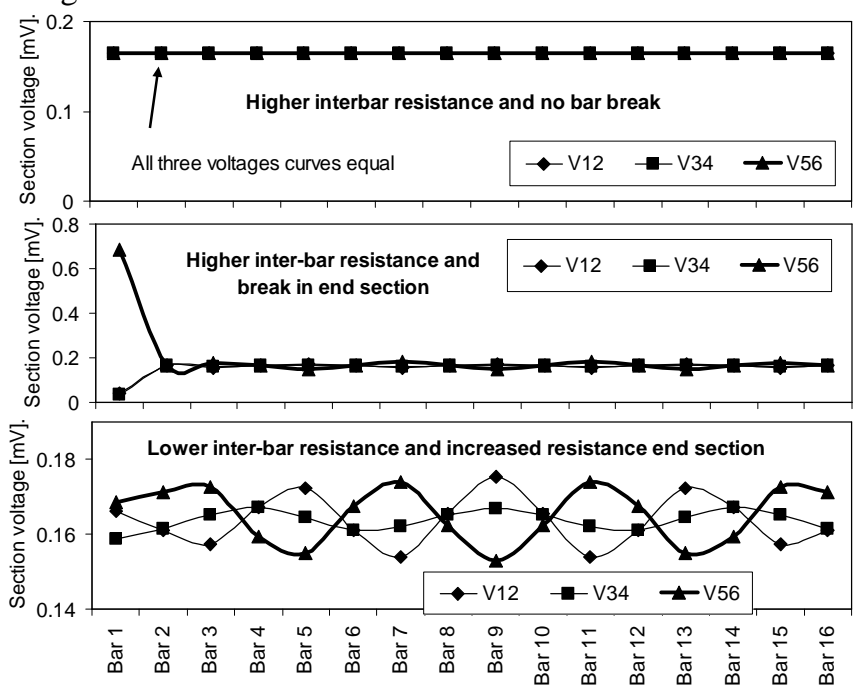

Fig. 20. Comparison of simulations results.

\section{CONCLUSIONS}

In this paper a set of experimental tests carried out on skewed cast copper cage rotors in three different conditions (healthy, with one broken bar and with all broken bars) have been reported and these highlight the influence of inter-bar current. The tests were carried out by injecting DC current into the rotor and measuring the voltage drop in five sections of equal length for each bar. The bar section voltages produced variation that may be follow characteristic patterns which indicate faults.

In addition a MATLAB simulation program was developed and this also indicated that in cast cage rotors, where there is low inter-bar resistance, there may be characteristic patterns in the section voltages when there are rotor faults.

While the results illustrate that inter-bar currents flow and that there are section voltage patterns, further investigation is needed to validate the patterns under a variety of faults in number of rotors. Initial results indicate that this patternrecognition method may be suitable for the development of a testing procedure for the detection of rotor cage anomalies after manufacture.

\section{REFERENCES}

[1] A.M. Odok, "Stray-load losses and stray torques in induction machines", Trans. AIEE, Part II, vol. 77, pp. 43-53, 1958.

[2] D.G. Dorrell, T.J.E. Miller, and C.B. Rasmussen, "Inter-bar currents in induction machines", IEEE Trans. Ind. Appl., vol. 39, no. 3, pp. $677-$ 684, May/Jun. 2003.

[3] K. Kerzenbaum, and C.F. Landy, "The existence of large inter-bar currents in three phase squirrel cage motors with rotor-bar and/or endring faults", IEEE Trans. on PAS, vol. 103, no. 7, pp.1854-1862, Jul. 1984.

[4] R.F. Walliser, and C.F. Landy, "Determination of interbar current effects in the detection of broken rotor bars in squirrel cage induction motors", IEEE Trans. Energy Conversion, vol. 9, no. 1, pp. 152-158, Mar. 1994.

[5] R.F. Walliser, and C.F. Landy, "Assessment of interbar current in double-cage induction motors with broken bars", IEEE Trans. Energy Conversion, vol. 9, no. 1, pp. 159-164, Mar. 1994.

[6] S. Williamson, R.C. Healey, J.D. Lloyd, and J.L. Tevaarwerk, "Rotor cage anomalies and unbalanced magnetic pull in single-phase induction motors", IEEE Trans. Ind. Appl., vol. 33, no. 6, pp. 1553-1562, Nov./Dec. 1997.

[7] P.J. Holik, and D.G. Dorrell, "Assessment of bar current in induction machines with broken bars using a multi-sliced finite element model", Proc. ICEM 2006, Crete, Greece, Sep. 2006.

[8] A. Bellini, C. Concari, G. Franceschini, C. Tassoni, and A. Toscani, "Vibrations, currents and stray flux signals to asses induction motors rotor conditions", Proc. IECON'2006, Paris, France, pp. 4963-4968.

[9] S. Williamson, and A.C. Smith, "Equivalent circuits for cage induction motors with inter-bar currents", IEE Proc. Electr Power Appl., vol. 149, no. 3, pp. 173-183, May 2002.

[10] L. Serrano-Iribarnegaray, and J. Martinez-Roman, "Critical review of the analytical approaches accounting for interbar currents and experimental study of ageing in two-speed asynchronous motors for elevator drives," IEE Proc.-Electr. Power Appl., vol. 152, no. 1, pp. 7280, Jan. 2005.

[11] S. Williamson, C.Y. Poh, and A.C. Sandy Smith, "Estimation of the Inter-Bar Resistance of a Cast Cage Rotor, IEEE Trans. Ind. Appl., vol. 40, no. 2, pp. 558-564, Mar./Apr. 2004.

[12] S. Williamson, and C.Y. Poh, "Inter-bar currents in cage induction motors", IEE Proc. Electr. Power Appl. vol. 152, no. 5, pp. 1106-1112, Sep. 2005.

[13] D.G. Dorrell, P.J. Holik, H.-J. Thougaard, F. Jensen, and P. Lombard, "Modelling axial variations in induction motors with rotor skew using multi-sliced 2D finite element analysis," in Proc. IEE PEMD Conf., 2006, pp. 686-690.

[14] D.G. Dorrell, P.J. Holik, and C.B. Rasmussen, "Analysis and effects of inter-bar current and skew on a long skewed-rotor induction motor for pump applications", IEEE Trans. Magn., vol. 43, no. 6, pp. 2534-2536, Jun. 2007.

[15] D.G. Dorrell, P.J. Holik, P. Lombard, H.-J. Thougaard, and F. Jensen, "A multi-sliced finite element model for induction machines incorporating inter-bar current", IEEE Trans. Ind. Appl., vol. 45, no. 1, pp. 131- 141, Jan/Feb 2009.

[16] J.J.C. Gyselinck, L. Vandevelde, and J.A.A. Melkebeek, “ Multi-slice FE modeling of electrical machines with skewed slots - The skew discretization error", IEEE Trans Magn., vol. 37, no. 5, pp. 3233-3237, Sep. 2001.

[17] R. Carlson, C. da Silva, N. Sadowski, Y. Lefevre, and M. LajoieMazenc, "Analysis of the effect of inter-bar currents on the performance of polyphase cage-induction motors," IEEE Trans. Ind. Appl., vol. 39, no. 6, pp. 1674-1680, Nov./Dec. 2003.

[18] A. Stening, C. Sadarangani, "The effect of inter-bar currents in aluminum and cast copper rotors", Proc. ICEM 2008, Vilamoura, Portugal, Sep. 2008. 